\title{
К ТЕОРИИ КОМБИНАЦИОННОГО РАССЕЯНИЯ СВЕТА С УЧЕТОМ КВАДРАТИЧНОГО ВИБРОННОГО ВЗАИМОДЕЙТВИЯ
}

1. В данной работе рассматривается метод преобразования спектров возбуждения резонансного комбинационного рассеяния (РКР), который основан на полученных в [ $\left.{ }^{1}\right]$ простых соотношениях между сечениями РКР и комплексным показателем преломлсния. С помощью указанных соотношений можно путем простой численной процедуры преобразовать спектр поглощения в пробные спектры возбуждения РКР (называемые возбуждающими профилями) и, сравнив их со спектрами, полученными в эксперименте, определить параметры вибронного взанмөдействия для всех активных в РКР колебаний $\left[{ }^{2}\right]$.

В разработанном к настояшему времени виде метод преобразования справедлив в приближении линейного вибронного и Герцберг-Теллеровского взаимодействий. Выполнен также ряд работ $\left[{ }^{3-9}\right]$, посвященных учету квадратичного взаимодействия, которое приводит к перепутыванию (вращению) системы нормальных координат колебаний при электронном переходе (т. н. вращение Душинского). Показано, что точный учет смешивания даже небольшого числа колебательных мод приводит к громоздким математическим выражениям, ввиду чего удается рассчитать эффекты перепутывания лишь небольшого числа мод при нулевой температуре.

В данной работе предлагается новый метод учета квадратичного вибронного взаимодействия, основанный на линейных соотношениях $\left[{ }^{10}\right]$ между фононными операторами в разных электронных состояниях. Он позволяет точно учесть вклад всех промежуточных состояний в сечение РКР. С помощью этого метода получены простые приближенные соотношения между поглощением и амплитудами РКР. Как и в случае линейного вибронного взаимодействия эти соотношения справедливы при произвольных числе мод и температуре. Они могут служить основой для обобщенного метода преобразования.

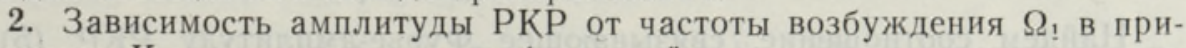
ближении Кондона определяется формулой

$$
\alpha_{i f}\left(\Omega_{1}\right)=C \sum_{m} \frac{\langle i \mid m\rangle\langle m \mid f\rangle}{E_{i}+\Omega_{1}-E_{m}-i \gamma},
$$

где $|i\rangle$ и $|f\rangle$ - начальное и конечное колебательное состояние в основном электронном состоянии, $|m\rangle$ - промежуточное колебательное состояние в возбужденном электронном состоянии, $\gamma$ - константа затухания последнего, $E_{i}$ и $E_{m}$ - энергии состояний $|i\rangle$ и $|m\rangle, C$ - нормировочная константа. Используя интегральное представление резольвенты, формула (1) может быть переписана в виде 
$\alpha_{i f}\left(\Omega_{1}\right)=i C \int_{0}^{\infty} d \tau \exp \left(-i \Omega_{1} \tau-\gamma \tau\right) \sum_{m}\left\langle i\left|\exp \left(-i \tau E_{i}\right) \exp \left(i \tau E_{m}\right)\right| m\right\rangle\langle m \mid f\rangle$.

Используя в (1а) соотношения $H_{1}|i\rangle=E_{i}|i\rangle, H_{2}|m\rangle=E_{m}|m\rangle\left(H_{\text {! и }} H_{2}\right.$ - колебательные гамильтонианы основного и возбужденного состояний) и условие полноты $\sum_{m}|m\rangle\langle m|=1$, получаем следующую формулу:

$$
\alpha_{i f}\left(\Omega_{1}\right)=C \int_{-\infty}^{\infty} d \tau \exp \left(-i \Omega_{1} \tau-\gamma \tau\right) \alpha_{i f}(\tau)
$$

где

$$
\alpha_{i f}(\tau)=i \theta(\tau)\left\langle i\left|\exp \left(-i \tau H_{1}\right) \exp \left(i \tau H_{2}\right)\right| f\right\rangle
$$

- нормированная Фурье-амплитуда PKР, $\theta(\tau)$ - ступенчатая функция Хэвисайда (она учитывает принцип причинности в РКР - рассеяние возникает после возбуждения). Фурье-амплитуда стоксова РКР первого порядка на колебании $i$ равна

$$
\alpha_{i}(\tau)=i \theta(\tau)\left(n_{i}+1\right)^{-1 / 2}\left\langle i\left|f_{\tau} a_{1 i}^{+}\right| i\right\rangle
$$

где

$$
f_{\tau}=\exp \left(-i \tau H_{1}\right) \exp \left(i \tau H_{2}\right)
$$

$n_{i}$ - номер исходного уровня колебания $i$.

Для нахождения сечения рассеяния при заданной температуре следует усреднить

$$
I_{i} \sim\left|\alpha_{i}\left(\Omega_{1}\right)\right|^{2} \sim \iint_{-\infty}^{\infty} d \tau d \tau^{\prime} \exp \left[i \Omega_{1}\left(\tau^{\prime}-\tau\right)-\gamma\left(\tau^{\prime}+\tau\right)\right] \alpha_{i}(\tau) \alpha_{i}\left(\tau^{\prime}\right)
$$

по исходным колебательным состояниям. Как показано в [ $\left.{ }^{11}\right]$, результат такого усреднения состоит в замене колебательных квантовых чисел на их температурные средние *. Поэтому при отличной от нуля температуре можно рассматривать усредненную по температуре Фурье-амплитуду, которая равна

$$
\alpha_{i(s)}(\tau)=\exp \left[-\omega_{1 i}(i \tau+1 / k T)\right] \alpha_{i(a)}(\tau)=i \theta(\tau)\left(\bar{n}_{i}+1\right)^{-1 / 2}\left\langle f_{\tau} a_{1 i}^{+}\right\rangle,
$$

где $(s)$ относится к стоксову, а $(a)-$ к антистоксову РКР,

$$
\begin{gathered}
\bar{n}_{i}=\left[\exp \left(\omega_{1 i} / k T\right)-1\right]^{-1}, \\
\langle\ldots\rangle=\mathrm{Sp}\left[\exp \left(-H_{1} / k T\right) \ldots\right] / \operatorname{Sp}\left[\exp \left(-H_{1} / k T\right)\right] .
\end{gathered}
$$

Здесь мы учли, что

$$
\left\langle f_{\tau} a_{1 i}\right\rangle=\left\langle f_{\tau} a_{1 i}^{+}\right\rangle \exp \left[-\omega_{1 i}(i \tau+1 / k T)\right] .
$$

Найдем соотношение, связывающее Фурье-амплитуду PKP (5) с Фурье-образом спектра поглощения

$$
F(\tau)=\left\langle f_{\tau}\right\rangle
$$

\footnotetext{
* Это утверждение справедливо как для кристаллических, так и для локальных колебаний, поскольку последние всегда могут быть рассмотрены как эйнштейновская зона кристаллических колебаний. Отличне локальных колебаний от кристаллических состонт в том, что для последних при $T \neq 0$ следует суммировать процессы с разным числом промежуточных фононов. В частности, для нахождения интенсивности $n$-фононной линии такого колебания, вообще говоря, следует просуммировать процессы $(n+2 k)$-го порядка для всех положительных целых, соответствующие рождению $n+k$ и уничтожению $k$ фононов. Однако учет таких процессов с виртуальными фононами существең лишь при весьма высоких температурах $\left[{ }^{10}\right]$.
} 
в модели квадратичного вибронного взаимодействия:

$$
V=H_{2}-H_{1}=V_{0}+(a q)+\frac{1}{2}(q b q) .
$$

Вектор $a$ и тензор $b$ описывают измснение сил $(a)$ и упругих постоянных $(b)$ колебательной системы при электронном переходе,

$$
q=\sum_{i} e_{1 i} x_{i}=\sum_{j} e_{2 j} y_{j}-q_{0}
$$

- вектор конфигурационных координат рассеивающего центра, $x_{i}$ и $y_{j}$ - нормальные координаты в основном и в возбужденном электронных состояниях,

$$
\begin{aligned}
& H_{1}=\frac{1}{2} \sum_{i}\left(-\frac{\partial^{2}}{\partial x_{i}^{2}}+\omega_{1 i}^{2} x_{i}^{2}\right) \\
& H_{2}=\frac{1}{2} \sum_{j}\left(-\frac{\partial^{2}}{\partial y_{j}^{2}}+\omega_{2 j}^{2} y_{j}^{2}\right)+\omega_{0},
\end{aligned}
$$

$\omega_{1 i}$ и $\omega_{2 j}$ - частоты нормальных колебаний, $\omega_{0}-$ частота чистоэлектронного перехода.

В модели (7) нормальные координаты $x_{i}$ и $y_{j}$ связаны ортогональным преобразованием вращения

$$
y_{j}=y_{0 j}+\sum_{i} c_{i j} x_{i}
$$

где

$$
c_{i j}=e_{1 i} b e_{2 j}\left(\omega_{2 j}^{2}-\omega_{1 i}^{2}\right)^{-1}
$$

- матрица вращения $\left[{ }^{12}\right], y_{0 j}=a e_{2 j} \omega_{2 j}^{-2}, \quad q_{0}=\sum_{j} e_{2 j} y_{0 j}, \quad e_{1 i}=\sum_{j} c_{i j} e_{2 j}$, $\sum_{i}\left(e_{1 i}^{2}\right)=\sum_{j}\left(e_{2 j}^{2}\right)=1$.

Учитывая, что $x_{i}=\left(a_{1 i}^{+}+a_{1 i}\right)\left(2 \omega_{1 i}\right)^{-1 / 2}, \quad \partial / \partial x_{i}=\left(a_{1 i}-a_{1 i}^{+}\right)\left(\omega_{1 i} / 2\right)^{1 / 2}$, $y_{j}=\left(a_{2 j}^{+}+a_{2 j}\right)\left(2 \omega_{2 j}\right)^{-1 / 2}, \quad \partial / \partial y_{j}=\left(a_{2 j}-a_{2 j}^{+}\right)\left(\omega_{2 j} / 2\right)^{1 / 2}$, получаем для операторов рождения фононов в разных электронных состояниях следующие соотношения:

$$
\begin{aligned}
& a_{1 i}^{+}=-\xi_{1 i}+\frac{1}{2} \sum_{j} \frac{c_{i j}}{\sqrt{\omega_{1 i} \omega_{2 j}}}\left[\left(\omega_{1 i}+\omega_{2 j}\right) a_{2 j}^{+}+\left(\omega_{1 i}-\omega_{2 j}\right) a_{2 j}\right], \\
& a_{2 j}^{+}=\xi_{2 j}+\frac{1}{2} \sum_{i} \frac{c_{i j}}{\sqrt{\omega_{1 i} \omega_{2 j}}}\left[\left(\omega_{1 i}+\omega_{2 j}\right) a_{1 i}^{+}-\left(\omega_{1 i}-\omega_{2 j}\right) a_{1 i}\right],
\end{aligned}
$$

где $\xi_{1 i}=\left(a+b q_{0}\right) e_{1 i}\left(2 \omega_{1 i}^{3}\right)^{-1 / 2}, \xi_{2 j}=a e_{2 j}\left(2 \omega_{2 j}^{3}\right)^{-1 / 2}$. (Соотношения для $a_{1 i}$ и $a_{2 j}$ получаются из (12) путем сопряжения.) Эти соотношения являются основой предлагаемого метода.

3. Рассмотрим коррелятор $\left\langle f_{\tau} a_{2 j}{ }^{+}\right\rangle$. Из соотношения (12) и формулы (6) следует

$$
\begin{gathered}
\left\langle f_{\tau} a_{2 j}^{+}\right\rangle=\xi_{2 j} F(\tau)+\frac{\sqrt{\omega_{2 j}}}{2} \sum_{k} \frac{c_{k j}}{\sqrt{\omega_{1 k}}}\left[1+\frac{\omega_{1 k}}{\omega_{2 j}}+\left(1-\frac{\omega_{1 k}}{\omega_{2 j}}\right) \exp \left(-\lambda \omega_{1 k}\right)\right] \times \\
\times\left\langle f_{\tau} a_{1 k}^{+}\right\rangle \quad(\lambda \equiv i \tau+1 / k T) .
\end{gathered}
$$


Переставим в левой части (13) операторы $\exp \left(i \tau H_{2}\right)$ и $a_{2 j}{ }^{+}$:

$$
\left\langle f_{\tau} a_{2 j}^{+}\right\rangle=\exp \left(i \tau \omega_{2 j}\right)\left\langle\exp \left(-i \tau H_{1}\right) a_{2 j}^{+} \exp \left(i \tau H_{2}\right)\right\rangle,
$$

выразим $a_{2 j}{ }^{+}$через $a_{1 k}$ и $a_{1 k}{ }^{+}$и учтем соотношения

$$
\begin{gathered}
\left\langle\exp \left(-i \tau H_{1}\right) a_{1 k} \exp \left(i \tau H_{2}\right)\right\rangle=\left\langle f_{\tau} a_{1 k}^{+}\right\rangle, \\
\left\langle\exp \left(-i \tau H_{1}\right) a_{1 k}^{+} \exp \left(i \tau H_{2}\right)\right\rangle=\exp \left(-\lambda \omega_{1 k}\right)\left\langle f_{\tau} a_{1 k}^{+}\right\rangle .
\end{gathered}
$$

Получим

$$
\begin{gathered}
\left\langle f_{\tau} a_{2 j}^{+}\right\rangle=\exp \left(i \tau \omega_{2 j}\right)\left\{\xi_{2 j} F(\tau)+\frac{\sqrt{\omega_{2 j}}}{2} \sum_{k} \frac{c_{k j}}{\sqrt{\omega_{1 k}}} \times\right. \\
\left.\times\left[\left(1+\frac{\omega_{1 k}}{\omega_{2 j}}\right) \exp \left(-\lambda \omega_{1 k}\right)+\left(1-\frac{\omega_{1 k}}{\omega_{2 j}}\right)\right]\left\langle f_{\tau} a_{1 k}^{+}\right\rangle\right\} .
\end{gathered}
$$

Умножая правые части (13) и (14) на $c_{i j} / \sqrt{\omega_{2 j}}$, суммируя по $j$ и учнтывая условие ортогональности

$$
\sum_{j} c_{i j} c_{k j}=\delta_{i k}
$$

получим

$$
\alpha_{i(s)}(\tau)=i \theta(\tau)\left(\bar{n}_{i}+1\right)^{-1 / 2}\left(2 \omega_{1 i}\right)^{1 / 2} p_{i}(\tau) F(\tau),
$$

где нормированная амплитуда $p_{i}(\tau)$ удовлетворяет уравнению

$$
\begin{gathered}
p_{i}=\beta_{i}+\sum_{k} R_{i k} p_{k}, \\
\beta_{i}=\frac{a}{2} \sum_{j} e_{2 j} c_{i j} \omega_{2 j}^{-1}\left(\exp \left(i \tau \omega_{2 j}\right)-1\right),
\end{gathered}
$$

$R_{i k}=\frac{1}{2} \sum_{j} c_{i j} c_{k j}\left\{\left(\frac{\omega_{2 j}}{\omega_{1 k}}-1\right)\left[\left(\bar{n}_{k}+1\right) \exp \left(i \tau \omega_{2 j}\right)-\bar{n}_{k} \exp \left(-i \tau \omega_{i k}\right)-1\right]+\right.$

$$
\left.+\bar{n}_{k}\left(\frac{\omega_{2 j}}{\omega_{1 k}}+1\right)\left[\exp \left(i \tau\left(\omega_{2 j}-\omega_{1 k}\right)\right)-1\right]\right\} \text {. }
$$

Отметим следующие свойства полученных выражений (16)-(19) для Фурье-амплитуды спектра возбуждәния РКР.

Фурье-амплитуда пропорциональна Фурье-образу спектра поглощения $F(\tau)$. Коэффициент пропорциональности состоит из константной и зависящей от времени $\tau$ частей. Последняя содержит лишь положительные степени экспонент $\exp \left(i \tau \omega_{2 j}\right)$ и отрицательные степени экспонент $\exp \left(i \tau \omega_{1 k}\right)$, причем последние фигурируют только в комбинации $\bar{n}_{k} \exp \left(-i \tau \omega_{1 k}\right)$. Учтем, что такими же свойствами обладает логарифм Фурье-образа спектра поглощения $\left[{ }^{10}\right]$. Поэтому в спектре поглощения содержатся лишь линии с рождением фононов с частотами возбужденного электронного состояния и с уничтожением фононов с частотами основного электронного состояния, причем последние пропорциональны числу таких фононов $\bar{n}_{i}$. Следовательно, в рассматриваемой модели спектр возбуждения РКР, рассматриваемый как функция частоты возбуждения $\Omega_{1}$, содержит резонансы только в тех частотах, что и спектр поглощения. Поскольку частоты колебаний в основном и в возбужденном состояниях разные, то в рассматриваемой модели РКР имеет точные резонансы по частоте возбуждения $\Omega_{1}$ и не имеет их по частоте 
рассеяния $\Omega_{2}=\Omega_{1}-\omega_{1 i}$ (имеются в виду резонансы с линиями спектра поглощения) .

4. Формулы (16)-(19) существенно упрощаются для малых времен $\tau$, когда можно разложить $\exp (i \tau \omega)$ в ряд и ограничиться первыми отличными от нуля членами. Это приближение законно, если резонансная полоса поглощения не имеет четкой колебательной структуры. Оно также всегда применимо при пререзонансном рассеянии.

В приближении малых времен $\beta_{i} \sim \tau$ и $R_{i k} \sim \tau$, поэтому в уравнении (17) справа можно пренебречь членом $\sim p_{k}$. При этом получим $p_{i} \simeq i \tau a e_{1 i} / 2$ и

$$
\alpha_{i(s)}(\tau)=\frac{a \tau \theta(\tau)}{\sqrt{2 \omega_{1 i}}}\left(\bar{n}_{i}+1\right)^{1 / 2} e_{1 i} F(\tau)
$$

Отсюда следует, что соотношение между профилем возбуждения PКР первого порядка $\left|\alpha_{i(s)}\left(\Omega_{1}\right)\right|^{2}$ и поглощением $x\left(\Omega_{1}\right)$ в случае пререзонансного возбуждения и при возбуждении в резонансе с бесструктурнсй полосой поглощения имеет вид

$$
\begin{gathered}
\left|\alpha_{i(s)}\left(\Omega_{1}\right)\right|^{2} \sim\left|\int_{-\infty}^{\infty} d \tau \exp \left(i \Omega_{1} \tau\right) \alpha_{i}(\tau)\right|^{2}= \\
=\frac{a^{2}}{2}\left(\bar{n}_{i}+1\right)\left|\frac{e_{1 i}}{\omega_{1 i}} \cdot \frac{d}{d \Omega_{1}} \Phi\left(\Omega_{1}\right)\right|^{2},
\end{gathered}
$$

где

$$
\Phi\left(\Omega_{1}\right)=i \pi x\left(\Omega_{1}\right)+\int_{-\infty}^{\infty} d x \frac{x(x)}{x-\Omega_{1}}
$$

- комплексный показатель преломления. Как видно, возбуждающий прсфиль РКР в данном случае определяется параметрами только ос но в ного электронного состояния: коэффициентом разложения линейного члена $a$ в адиабатическом потенциале $V$, компонентами $\left(e_{1 i}\right)$ вектора $q$ в пространстве нормальных координат $x_{i}$ и частотой $\left(\omega_{1 i}\right)$ моды $i$.

Отношение интенсивностей рассеяния основного тона на разных модах $i$ и $i^{\prime}$, согласно (21), равно

$$
\frac{I_{1 i}}{I_{1 i^{\prime}}}=\frac{e_{1 i}^{2}\left(\bar{n}_{i}+1\right)}{e_{1 i^{\prime}}^{2}\left(\bar{n}_{i^{\prime}}+1\right)} \cdot \frac{\omega_{1 i^{\prime}}}{\omega_{1 i}}=\frac{\left(\xi_{1 i} \omega_{1 i}\right)^{2}}{\left(\xi_{1 i^{\prime}} \omega_{1 i^{\prime}}\right)^{2}} \cdot \frac{\bar{n}_{i}+1}{\bar{n}_{i^{\prime}}+1}
$$

Видим, что с учетом квадратичного взаимодействия в пререзонансном рассеянии (также как и в резонансном при бесструктурной полосе поглощения) остается в силе формула Савина, полученная в $\left[{ }^{13}\right]$ для модели линейного вибронного взаимодействия. Аналогичный результат при $T=0$ получен Хеллером $\left[{ }^{12}\right]$.

5. Во многих случаях хорошо выполняется приближение $|b| \bar{\omega}^{-2} \ll 1$ $(\vec{\omega}-$ средкяя частота колебаний $)$, означающее, что эффекты перепутывания (если они есть) существенны лишь для колебаний $i$ и $j$ близких частот $\left(\left|\omega_{1 i}-\omega_{2 j}\right| \ll \omega_{1 i}\right)$. В этом приближении при низких температурах $p_{i} \simeq p_{i}{ }^{(0)}=\beta_{i}$ и

$$
\alpha_{i(s)}^{(0)}(\tau)=\frac{i \theta(\tau)\left(\bar{n}_{i}+1\right)^{1 / 2}}{\sqrt{2 \omega_{1 i}}} \sum_{j} \frac{c_{i j} e_{2 j}}{\omega_{2 j}}\left(\exp \left(i \tau \omega_{2 j}\right)-1\right) F(\tau)
$$


Действительно, по порядку величины $\left|\beta_{i}\right| \leqslant\left|a e_{1 i} \omega_{1 i}^{-1}\right|, \quad c_{k j}\left(1-\frac{\omega_{2 j}}{\omega_{1 k}}\right)<$ $<\left|b e_{1 k} e_{2 j}\right| \omega_{1 k}^{-2}$. Поэтому при низких температурах $R_{i k}<\left|b e_{1 i} e_{1 k}\right| \omega_{1 k}^{-2}$ и первая поправка к решению $p_{i}^{\left({ }^{(0)}\right.} \simeq \beta_{i}$ равна $p_{i}^{(1)} \leqslant\left|b \beta_{i}\right| \sum_{k} e_{1 k}^{2} \omega_{1 h}^{-2} \sim$ $\sim\left|\beta_{i} b\right| \vec{\omega}^{-2} \ll\left|\beta_{i}\right|$.

Фурье-преобразование формулы (24) дает следующее соотношение между амплитудой РКР и комплексным показателем преломления:

$$
\alpha_{i(s)}\left(\Omega_{1}\right) \simeq\left(\bar{n}_{i}+1\right)^{1 / 2} \frac{a}{\sqrt{2 \omega_{1 i}^{3}}}\left[\bar{e}_{1 i} \Phi\left(\Omega_{1}\right)-\sum_{j} \bar{c}_{i j} e_{2 j} \Phi\left(\Omega_{1}-\omega_{2 j}\right)\right],
$$

где $\bar{c}_{i j}=c_{i j}\left(\omega_{1 i} / \omega_{2 j}\right), \quad \bar{e}_{1 i}=\sum_{j} \bar{c}_{i j} e_{2 j} \approx e_{1 i}$.

Эта формула обобщает формулу линейного взаимодействия [1]

$$
\alpha_{i(s)}\left(\Omega_{1}\right)=\left(\bar{n}_{i}+1\right)^{1 / 2} \xi_{1 i}\left[\Phi\left(\Omega_{1}\right)-\Phi\left(\Omega_{1}-\omega_{1 i}\right)\right] .
$$

Формула (25) справедлива при произвольном смешивании (произвольных $c_{i j}$ ) колебаний $i$ и $j$ с близкими частотами и при слабом смешивании колебаний с существенно разными частотами. Эта формула по своей структуре аналогична (26). Поэтому она позволяет, в принципе, столь же просто определять параметры смешивания $c_{i j}$, как и параметры линейного вибронного взаимодействия $e_{1 i}$.

Если значительная часть вибронного взаимодействия обусловлена низкочастотными (акустическими) колебаниями, то в (19) даже при низкой температуре следует учитывать связанные с этими колебаниями члены $\sim \bar{n}_{k}$. Отмеченный вклад в $R_{i k}$ низкочастотных колебаний имеет вид

$$
R_{i k}^{(1)} \simeq \frac{\bar{n}_{k}}{2 \omega_{1 i}^{2} \omega_{1 k}} \sum_{j} \frac{e_{1 i} b e_{2 j} e_{2 j} b e_{1 k}}{\omega_{2 j}-\omega_{1 k}}\left[\exp \left(i \tau\left(\omega_{2 j}-\omega_{1 k}\right)\right)-1\right] .
$$

Учтем, что структурная часть возбуждающего профиля РКР связана с незатухаюей частью $\alpha_{i}(\tau)$ при больших $\tau$. В области таких $\tau$ можно $\exp \left[i \tau\left(\omega_{2 j}-\omega_{1 k}\right)-1\right]\left(\omega_{2 j}-\omega_{1 k}\right)^{-1}$ заменить на $i \pi \delta\left(\omega_{2 j}-\omega_{1 k}\right)$. Тогда

$$
R_{i k}^{(1)} \simeq i \pi \bar{n}_{k} \omega_{1 i}^{-2}\left(e_{1 i} b \operatorname{Im} G_{2}\left(\omega_{1 k}\right) b e_{1 k}\right),
$$

где

$$
G_{2}(\omega)=\sum_{j} \frac{e_{2 j} e_{2 j}}{\omega^{2}-\omega_{2 j}^{2}-i \varepsilon \omega}, \quad \varepsilon \rightarrow 0,
$$

- колебательная функция Грина возбужденного электронного состояния. Подставляя (28) в (17) и пренебрегая быстро затухающей при больших $\tau$ поправкой, получаем снова формулу (25), в которой действительный параметр $\bar{e}_{1 i}$ заменяется на комплекснєй $\bar{e}_{1 i}+i \zeta_{i}$, где

$$
\zeta_{i}=\pi \omega_{1 i}^{-1} b \sum_{k} \bar{n}_{k} \omega_{1 k}^{-1} e_{1 k} b \operatorname{Im} G_{2}\left(\omega_{1 k}\right) \bar{e}_{1 k}
$$

(в случае дебаевской модели

$$
G_{2}(\omega) \sim \omega^{3} / 5 \omega_{D}^{5} \text { и } \zeta_{i} \sim 0,1 b^{2}(k T)^{3} \omega_{D}^{-5},
$$
где $\omega_{D}-$ дебаевская частота). Это означает, что смешивание с непрерывной ветвью колебаний изменяет фазу парциальной амплитуды РКР $\sim \Phi\left(\Omega_{1}\right)$. Указанное изменение фазы существенно для интерференции разных каналов комбинационного рассеяния (KР) (например, резонансного КР с нерезонансным, имеющем действительную амплитуду). 
При сильном вибронном взаимодействии с несколькими локальными колебательными модами спектр поглощения может иметь четкую колебательную структуру и тогда, когда $|b| \bar{\omega}^{-2} \geqslant 1$. В этом случае в первом приближении в уравнении (17) можно учесть лишь $p_{k}$, относящиеся к этим колебаниям, а для всех остальных колебаний использовать рассмотренное выше приближение $p_{i}{ }^{(0)} \simeq \beta_{i}$. Уравнение (17) для отмеченных нескольких колебаний может быть легко решено численно. Полученное таким способом приближенное решение может быть уточнено с использованием обычной итерационной процедуры.

6. В резонансном и (особенно) в пререзонансном случае существенно учитывать также Герцберг-Теллеровское взаимодействие, приводящее к отклонению от приближения Кондона. В этом случае

$$
\begin{gathered}
F(\tau)=\left\langle\exp \left(-i \tau H_{1}\right) M \exp \left(i \tau H_{2}\right) M\right\rangle, \\
\alpha_{i(s)}(\tau)=i \theta(\tau)\left(\bar{n}_{i}+1\right)^{-1 / 2}\left\langle\exp \left(-i \tau H_{1}\right) M \exp \left(i \tau H_{2}\right) M a_{1 i}^{+}\right\rangle,
\end{gathered}
$$

где $M$ - электронный матричный элемент. В линейном приближении

$$
M \simeq 1+\sum_{k} m_{k}\left(a_{1 k}^{+}+a_{1 k}\right),
$$

связанная с этим взаимодействием поправка к Фурье-амплитуде (16) имеет вид

$$
\alpha_{i(s)}^{\neq c}(\tau) \simeq \sum_{k} m_{k}\left\langle f \tau\left[a_{1 i}^{+}\left(a_{1 k} \exp \left(\lambda \omega_{1 k}\right)+a_{1 k}^{+} \exp \left(-\lambda \omega_{1 k}\right)\right)+\left(a_{1 k}^{+}+a_{1 k}\right) a_{1 i}^{+}\right]\right\rangle
$$

При малом отклонении от приближения Кондона основной вклад в $\alpha_{i} \neq c$ дает спаривание операторов $a_{1 k}$ и $a_{1 i}{ }^{+}$. Остальные спаривания (они рассмотрены в $\left.\left[{ }^{10,14}\right]\right)$ приводят к членам $\sim m_{1 k} \xi_{1 i} \xi_{1 k}$, которые учитывают перенормировку Фурье-образа спектра поглощения, связанную с отклонением от приближения Кондона, и к малым членам $\sim|b| \bar{\omega}^{-2} \sum_{k} m_{k} e_{1 k} . \quad$ Таким образом, в первом приближении

$$
\alpha_{i(s)}^{\neq C}(\tau)=i \theta(\tau) m_{i}\left(\bar{n}_{i}+1\right)^{1 / 2}\left(1+\exp \left(i \tau \omega_{1 i}\right)\right) F(\tau)
$$

и

$$
\alpha_{i(s)}^{\neq c}\left(\Omega_{1}\right)=m_{i}\left(\bar{n}_{i}+1\right)^{1 / 2}\left[\Phi\left(\Omega_{1}\right)+\Phi\left(\Omega_{1}-\omega_{1 i}\right)\right]
$$

Из этой формулы следует, что $\operatorname{Re} \alpha_{i(s)}^{\neq c}\left(\Omega_{1}\right)$ имеет максимумы в частотах возбуждения $\Omega_{1}$ и рассеяния $\Omega_{2}=\Omega_{1}-\omega_{1 i}$, совпадающие с максимумами спектра поглощения. Следовательно, в случае квадратичного вибронного взаимодействия отклонение от приближения Кондона восстанавливает в возбуждающем профиле РКР резонансы по рассеянному свету.

7. В заключение отметим, что метод преобразования возбуждающих профилей РКР, как было показано выше, позволяет одновременно учесть смещения положений равновесия и перепутывание нормальных координат при электронном переходе (вращение Душинского), а также отклонение от приближения Кондона. В наиболее актуальном случае значительного смешивания мод близких частот (и малого смешивания мод сушественно разных частот) основные соотношения метода (см. формулы (24) и (25)) сохраняют простүю структуру, характерную для линейного вибронного взаимодействия. Поэтому они позволяют, в принципе, столь же просто определять параметры смешивания мод, как и параметры линейных вибронного и Герцберг-Теллеровского взаимодействий. 
Наиболее важным качественным отличием возб́уждающих профилей РКР в модели квадратичного вибронного взаимодействия от линейного является исчезновение в приближении Кондона резонансов по рассеянному свету; однако они восстанавливаются при одновременном учете квадратичного вибронного и Герцберг-Теллеровского взаимодействий.

\section{ЛИТ Е Р А Т У Р А}

1. Hizhnyakov, V., Tehver, I. Phys, status solidi, 21, № 2, 755-768 (1967).

2. Champion, P. M., Albrecht, A. C. Ann. Rev. Phys. Chem., 33, 353-376 (1982); Brafman, O., Chan, C. K., Khodadoost, B., Page, J. B., Walker, C. T. J. Chem. Phys., 80, № 11, 5406-5417 (1984).

3. Hizhnyakov, V. V., Tehver, I. J. Opt. Commun., 32, № 3, 419-421 (1980).

4. Tonks, D. L., Page, J. B. Chem. Phys. Lett., 79, № 2, 247-252 (1981).

5. Siebrand, W., Zgierski, M. J. Chem. Phvs., 71, № 9, 3561-3569 (1979).

6. Jean, J. M., Friesner, R. A. J. Chem. Phys., 85, № 5, 2353-2364 (1986).

7. Kupka, H., Cribb, P. H. J. Chem. Phys., 85, № 3, $1303-1315$ (1986).

8. Sue, J., Xan, Y. J., Mukamel, S. J. Chem. Phys., 85, № 1, $462-474$ (1986).

9. Tannor, D. J., Heller, E. J. J. Chem. Phvs., 77, № 1, 202-212 (1982).

10. Хижняков В. В. Тр. ИФ АН ЭССР, № 59, 55-74 (1986).

11. Хижняков В. В. Тр. ИФ АН ЭССР, № 32, 16-30 (1967).

12. Heller, E. J., Sundberg, R. L., Tannor, D. J. J. Phys. Chem., 86, $1822-1833$ (1982).

13. Савин Ф. А. Опт. и спектр., 19, вып. 4, 555-561 (1965).

14. Hizhnyakov, V. V. Phys. status solidi (b), 114, № 2, 721-730 (1982).

Ннститут физики

Академии наук Эстонской ССР
Поступила в редакцию 1/IV 1987

\section{HIZ̃NJAKOV, Imbi TEHVER \\ VALGUSE KOMBINATSIOONHAJUMISE TEOORIAST BILINEAARSE VIBROONINTERAKTSIOONI KORRAL}

On esitatud uus meetod bilineaarse vibrooninteraktsiooni arvestamiseks valguse kombinatsioonhajumise amplituudide (ergastusprofiilide) arvutamisel. Meetod pōhineb lineaarsetel seostel, mis seovad foononoperaatoreid erinevates elektronseisundeis. On saadud valemid, mis seovad hajumisamplituude neeldumisspektriga (teisendusmeetodi üldistus). Järeldub, et bilineaarne vibrooninteratsioon tingib resonantsi kadumise hajumissageduse järgi, mis taastub, kui samaaegselt arvestada ka Herzberg-Telleri interaktsiooni.

\section{HIZHNYAKOV, Imbi TEHVER \\ ON THE THEORY OF RAMAN SCATTERING WITH QUADRATIC VIBRONIC COUPLING}

A new method for considering the quadratic vibronic coupling in the Raman amplitude (excitation profile) calculations is proposed. The method is based on the linear transformation between phonon operators in different electronic states. Formulas have been obtained that express the Raman amplitudes via the absorption spectrum (the generalization of the transform method). It is shown that the quadratic vibronic coupling leads to the vanishing of the resonance in scattered light. However, it will be restored when the Herzberg-Teller interaction is taken into account simultaneously. 